\title{
Analysis of Cubic Boron Nitride Single Crystal Defects Growth under High Temperature and High Pressure
}

\author{
Lichao Cai, ${ }^{1}$ Bin Xu, ${ }^{2}$ Meizhe $\mathrm{Lv} \mathbb{D D}^{1}{ }^{1}$ Feng Jia, ${ }^{2}$ and Xingdong Yuan ${ }^{2}$ \\ ${ }^{1}$ School of Materials Science and Engineering, Shandong University, Jinan 250101, China \\ ${ }^{2}$ School of Materials Science and Engineering, Shandong Jianzhu University, Jinan 250101, China \\ Correspondence should be addressed to Meizhe Lv; lvmeizhe19@sdjzu.edu.cn
}

Received 11 July 2020; Accepted 3 August 2020; Published 24 August 2020

Guest Editor: Tifeng Jiao

Copyright (c) 2020 Lichao Cai et al. This is an open access article distributed under the Creative Commons Attribution License, which permits unrestricted use, distribution, and reproduction in any medium, provided the original work is properly cited.

Cubic boron nitride $(\mathrm{cBN})$ single crystals are synthesized under high temperature and high pressure in the Li-based system. The growth defects on hexagonal and triangular (111) surfaces of cBN single crystals after rapid cooling are discussed systemically for the first time using the atomic force microscope. Some impurity particles, triangle cone hole defects, lamellar-fault structures, and big steps are obvious on the surfaces of $\mathrm{cBN}$ single crystals. The formation mechanism of these defects is analyzed briefly at the synthetic process of $\mathrm{cBN}$ single crystals, and the growth mechanism of $\mathrm{cBN}$ single crystals transform from the two-dimensional growth to dislocation growth mechanism under high temperature and high pressure.

\section{Introduction}

Cubic boron nitride ( $\mathrm{cBN}$ ) single crystals have a high hardness, high melting point, high thermal conductivity, wide energy gap, and low dielectric constant, which make them highly promising materials [1-4]. At present, cBN single crystals are synthesized using hexagonal boron nitride $(\mathrm{hBN})$ as the source material and lithium nitride $\left(\mathrm{Li}_{3} \mathrm{~N}\right)$ as the catalyst by the static high temperature and high pressure (HPHT) catalytic method [5-7]. The further development of electronic and optical applications need large $\mathrm{cBN}$ single crystals with high quality, while the size of present $c B N$ single crystals cannot meet the demand for the study of advanced functional products $[8,9]$. In a previous experimental study, the cBN nucleation was discussed, which indicates that $\mathrm{cBN}$ crystal nuclei are formed directly by the transition of $\mathrm{hBN}$ [10]. The growth process of $\mathrm{cBN}$ single crystals determines their quality after $\mathrm{cBN}$ nucleation under HPHT. The growth defects could affect the crystal quality; thus, the importance of growth defects in cBN single crystals growth should be clearly recognized [11]. It is almost impossible to study in situ the growth process of cBN single crystals under HPHT; however, much information about cBN growth under HPHT is preserved on the cBN crystal surfaces and the interface layer of $\mathrm{cBN}$ single crystals at room temperature and ambient pressure when the synthetic cell assembly was cooled rapidly. The morphology of crystals surfaces and the constituent of the interface layer are closely associated with the $\mathrm{cBN}$ single crystals growth under HPHT, and it may be of great significance to explain the growth mechanism of cBN single crystals.

At present, the study of crystal growth at nanometer sale has attracted much interest, and some new growth theories have been established with the help of the AFM [11]. The surface topography of synthetic $\mathrm{cBN}$ single crystals was reported $[4,10,12]$; however, thus far, there is almost no report on the analysis of $\mathrm{cBN}$ single crystal surfaces by atomic force microscopy (AFM). The factors that affect the formation of defects on (111) surfaces of cBN single crystals and the growth mechanism of $\mathrm{cBN}$ single crystals are 


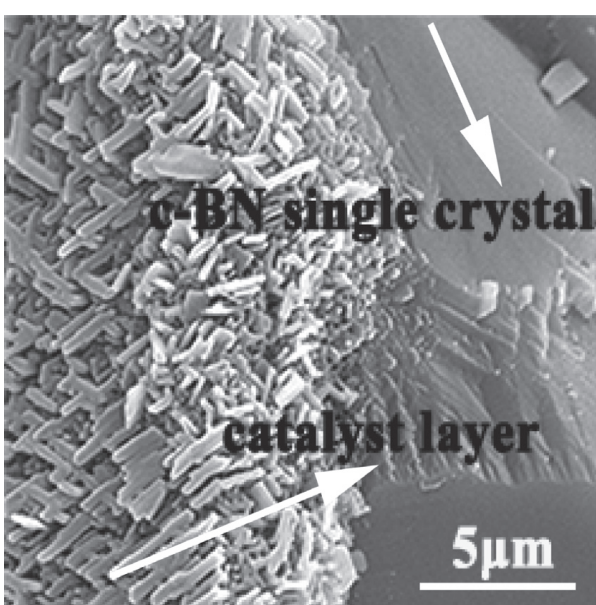

(a)

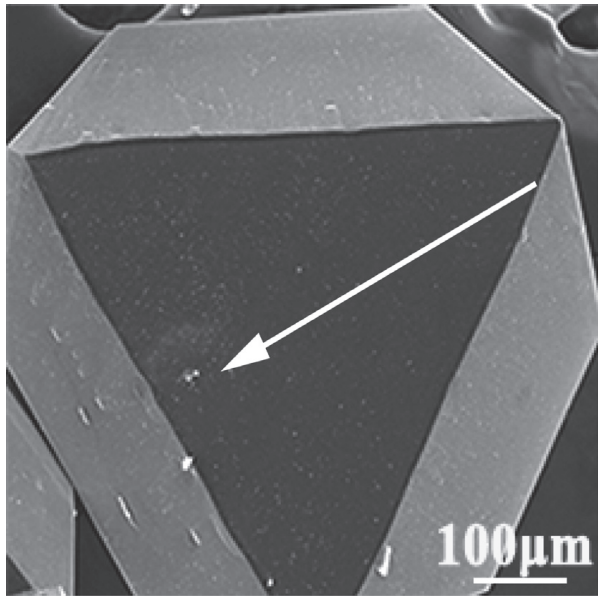

(c)

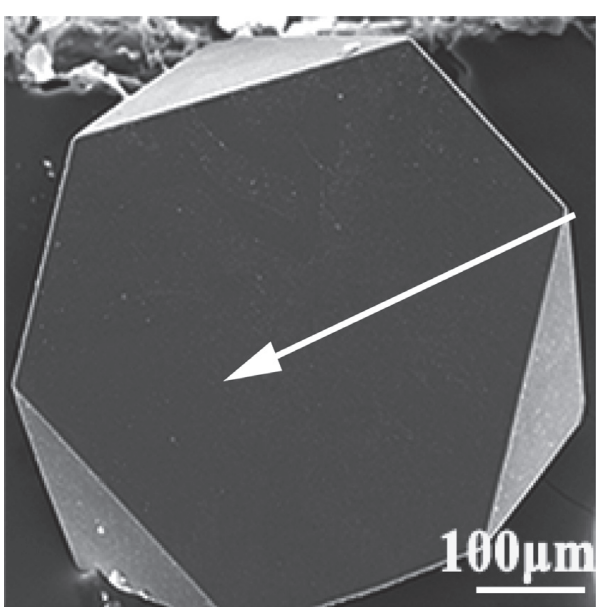

(b)

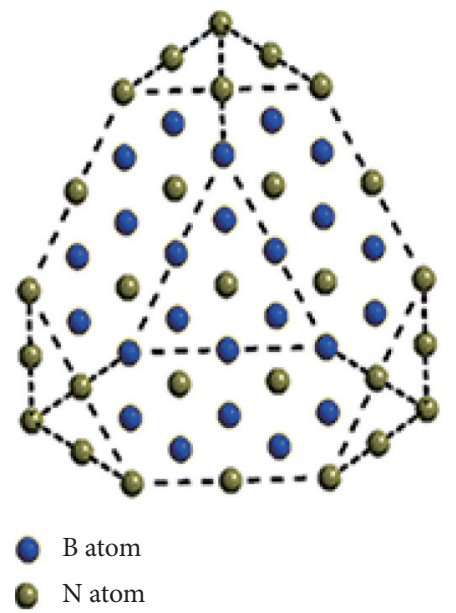

(d)

Figure 1: (a) SEM images of cBN single crystals surrounded by the powders in the interface layer. (b, c) cBN with hexagonal and triangular (111) surfaces. (d) Plane projection drawing of a cBN single crystal.

discussed on the basis of the AFM observations. The present study provides a better understanding of $\mathrm{cBN}$ single crystals growth mechanism under HPHT.

\section{Materials and Methods}

cBN single crystals were synthesized from high purity hBN (>98\%) with $\mathrm{Li}_{3} \mathrm{~N}$ as catalyst, and the weight ratio of the two was 9:1 by the static HPHT catalyst method. HPHT experiments were performed using the cubic anvil apparatus HTDS-034 hm. In these experiments, the synthetic pressure, temperature, and time were $4.5 \mathrm{GPa}, 1700 \mathrm{~K}$, and $10 \mathrm{~min}$, respectively. After rapid cooling, the synthesized $\mathrm{cBN}$ bulk was removed from the cubic anvil and prepared for further examination. As shown in Figure 1(a), the cBN single crystal was surrounded by powders. The samples were cleaned, and then, the images of $\mathrm{cBN}$ single crystals were taken using a JSM-6380 LA-type scanning electron microscope (SEM). The morphology of HPHT as-grown crystal surfaces was obtained using a Bruker Multimode 3D AFM in contact mode under ambient atmosphere and room temperature.
The powders around the HPHT as-grown cBN single crystals were carefully collected under the view of an optic microscope. A JEOLJEM-2010F-type high resolution transmission electron microscope (HRTEM) was used to examine their phases in powders with an operating voltage of $200 \mathrm{kV}$.

\section{Results and Discussion}

Since the facets are in direct contact with the molten catalyst under HPHT in the $\mathrm{CBN}$ crystal growth process, the surface morphology provides much information to analyze the crystal growth. Figures 1(b) and 1(c) shows the SEM images of $\mathrm{cBN}$ single crystals with triangular and hexagonal facets (111) and their plane projection drawing. The sizes of $\mathrm{cBN}$ single crystals are about $0.5 \mathrm{~mm}$. The synthesized $\mathrm{cBN}$ single crystals have truncated tetrahedron structures, which are composed of four coordination $\mathrm{B}-\mathrm{N}_{4}$ or $\mathrm{N}-\mathrm{B}_{4}$ growth units. There exist solid structures in short or medium range order in the molten catalyst when cBN single crystals are synthesized under HPHT [10, 12]; thus, much information about the molten catalyst can be remained in the powders 


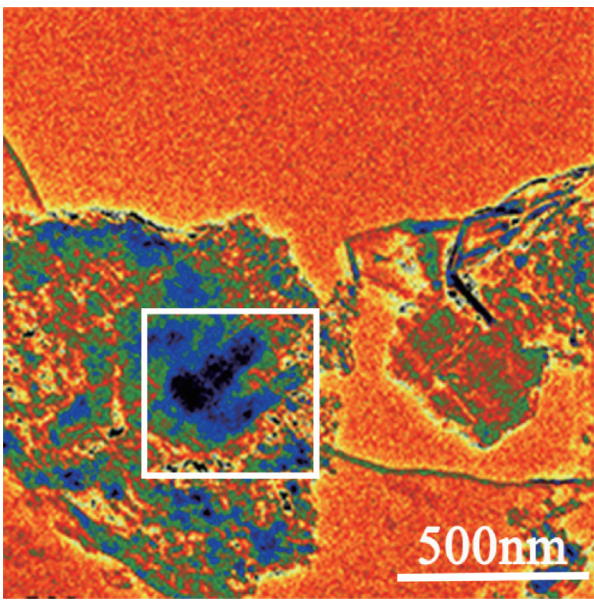

(a)

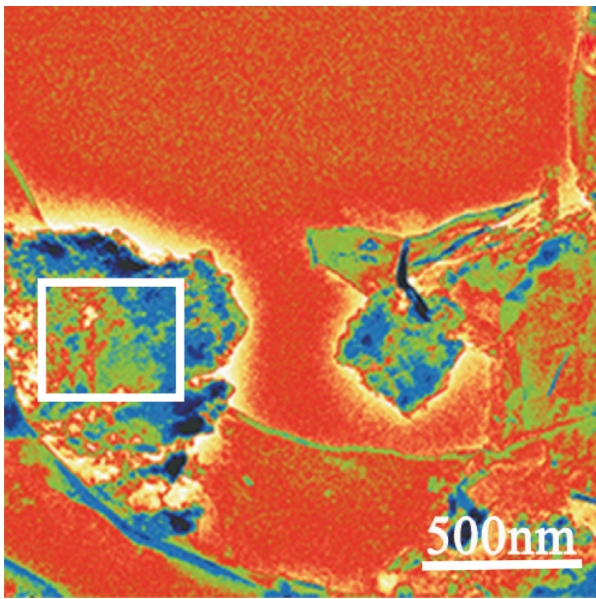

(c)

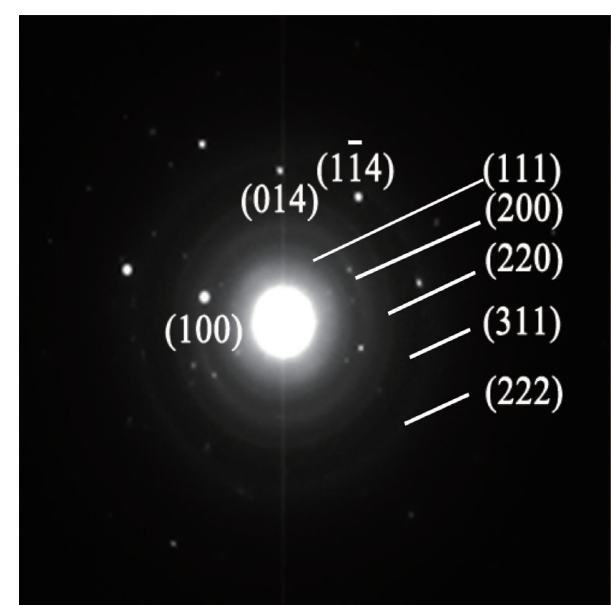

(b)

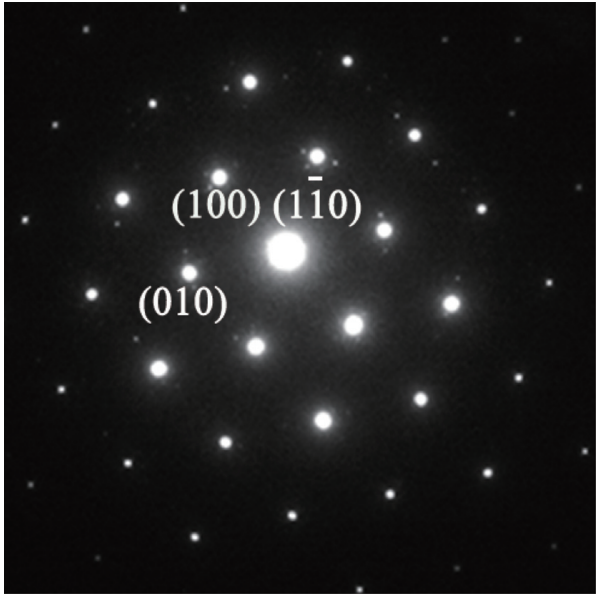

(d)

FIgURE 2: (a) The morphology of powders surrounded cBN single crystals. (b) The corresponding SAD pattern in the white grid of (a). (c) The morphology at same position as (a) after irradiated by HRTEM. (d) The corresponding SAD pattern in the white grid of (c).

surrounded by cBN single crystals at ambient condition after the cell assembly is cooled rapidly.

The powders in the interface layer (in Figure 1(a)) were collected, and their compositions were determined with HRTEM. Figures 2(b) and 2(d) show the SAD patterns of powders surrounded $\mathrm{cBN}$ single crystals in Figures $2(\mathrm{a})$ and 2(c), respectively. The corresponding SAD pattern in Figure 2(b) indicates that the white powders in Figure 2(a) are $\mathrm{cBN}$ and $\mathrm{hBN}$, from which $\mathrm{cBN}$ can be identified by (111), (200), (220), (311), and (222) diffraction ring, while hBN can be identified by (100), (014), and (11 4) diffraction pattern. This means many microcubic grains are formed under HPHT in the growth process of $\mathrm{cBN}$ crystals. However, the corresponding SAD pattern in Figure 2(d) indicates that the white powders in Figure 2(c) are all hBN, from which hBN can be identified by (100), (010), and (110) diffraction pattern. Figures 2(a) and 2(c) are the morphologies of the same zone of the same powders before and after electron beam irradiation. It is obvious that the black material in Figure 2(a) disappears.

This means the bunching electron beam by HRTEM induces the occurrence of the reaction from $c B N$ to $h B N$.
From Figure 2(b), it can be inferred that it is a good corresponding relation between (200) of cBN and (100) of hBN crystal planes. The $\mathrm{cBN}$ growth process suggests that when $\mathrm{hBN}$ transform into micro-cBN grain under HPHT, various micro-cBN grains are delivered to the growing single crystal surfaces, and the collision or coalescence of micro-cBN grains in the molten catalyst induces the growth of $\mathrm{cBN}$ single crystals under HPHT. Meanwhile, it is possible that the recrystallization of $\mathrm{hBN}$ exists in HPHT experiments. This suggests are consistent with the previous experimental results that $\mathrm{cBN}$ with higher quality is synthesized under $4.5 \mathrm{GPa}$ and $1800 \mathrm{~K}$ [13]. In our earlier work, $\mathrm{Li}_{3} \mathrm{BN}_{2}$ are found in the interface by meaning of XRD, and it is the real catalyst $[10,12]$. The crystal surface energy can directly affect the nucleation and growth of the crystal. $\mathrm{Li}_{3} \mathrm{BN}_{2}$ has higher surface energy [14]; thus, the $\mathrm{Li}_{3} \mathrm{BN}_{2}$ phase can restrain the crystals' nucleation at the initial stage of the reaction and then promote the maximum growth of the nucleus under high temperature and low pressure.

Figure 3 shows different growth defects on the surfaces of $\mathrm{cBN}$ single crystals. Although $\mathrm{cBN}$ crystal surfaces were cleaned, some microscopic particles were still covered on the 

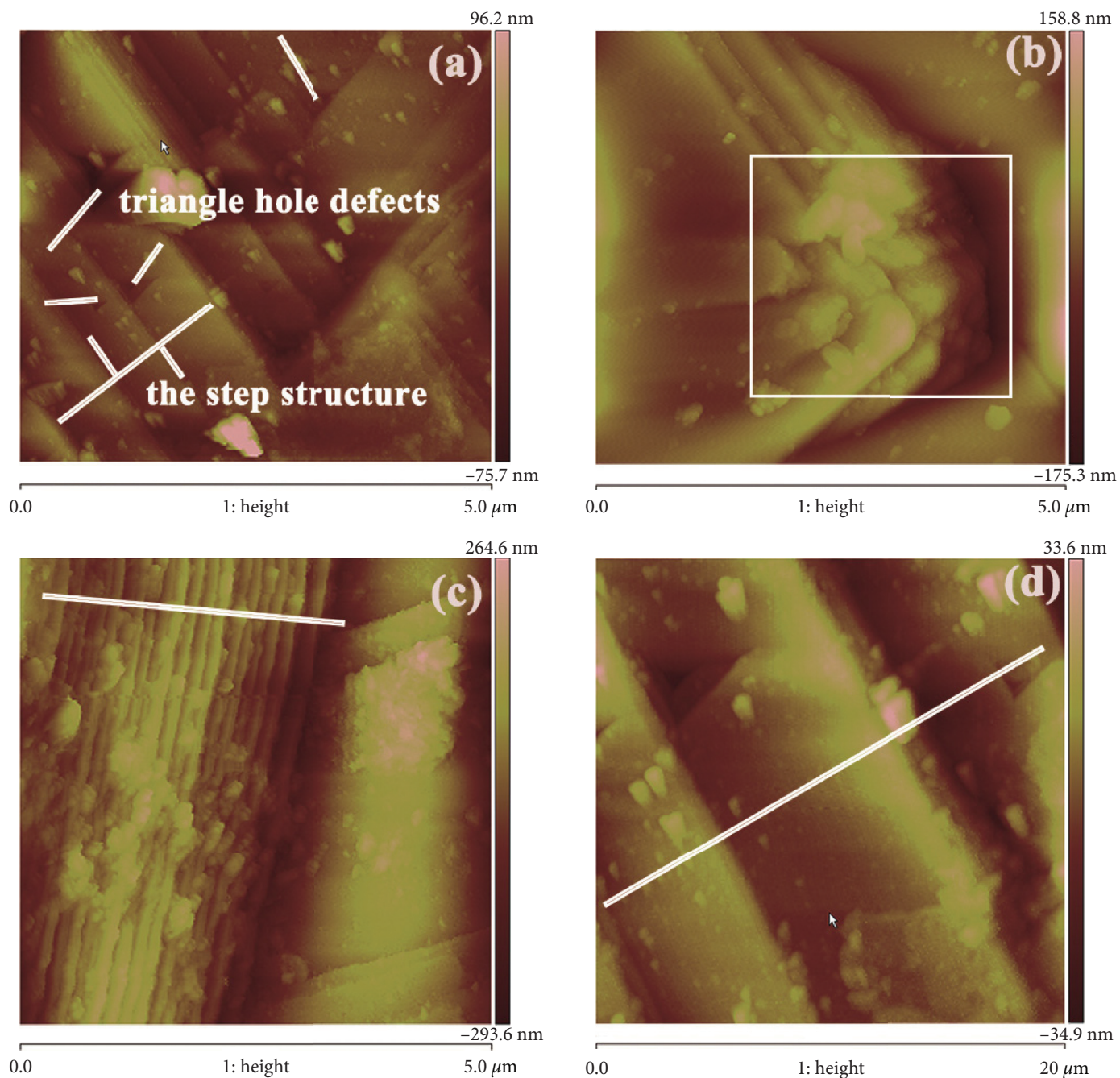

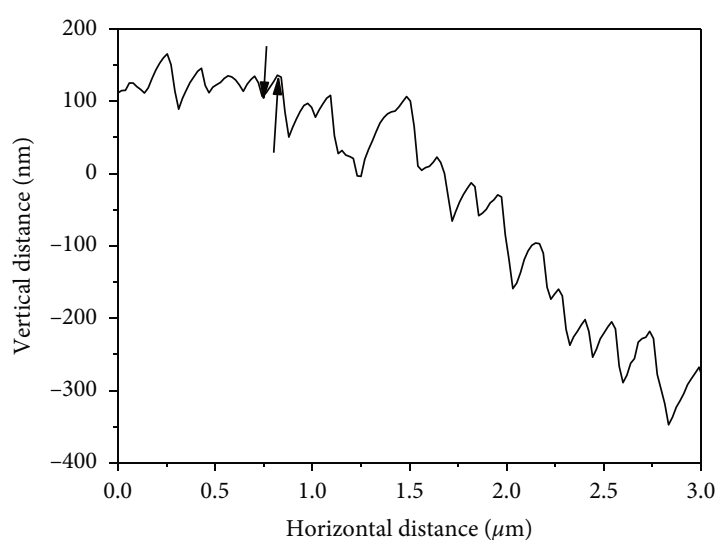

(e)

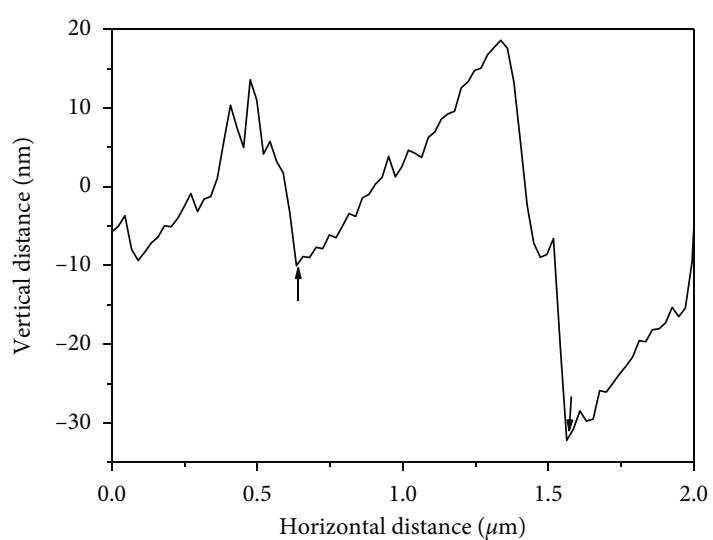

(f)

Figure 3: (a) Microparticles on (111) facets, (b) the lamellar-fault structure on (111) facets, and (c) the big step structures and triangle-hole defects on (111) facets. (d) The amplification image of area A in (c), (e), and (f) section analyses suggest the height of two steps shown by arrows in (b) and (c), respectively.

single crystal surfaces, as shown in Figure 3(a). Microscopic particles were probably derived from impurities adhering on the crystal surfaces or remaining powders surrounding $\mathrm{cBN}$ single crystals. Meanwhile, triangular hole defects, lamellarfault structures, and big step structures are found in
Figure 3(a). Figures 3(c) and 3(d) show the 2D AFM images of lamellar-fault structures and big step structures on cBN crystal (111) facets in an area of $5 \mu \mathrm{m} \times 5 \mu \mathrm{m}$, respectively. From section analysis in Figure 3(e), it was found that the height of a lamellar demonstrated by an arrow in Figure 3(c) 
is about $27 \mathrm{~nm}$, and the height of the lamellar-fault structures has relatively homogeneous distribution. The height of a big step in Figure 3(d) is about $28 \mathrm{~nm}$, which is basically the same as the height of the lamellar-fault structures. Compared to the height of lamellar-fault structures in Figure 3(c), the big step structures in Figure 3(d) have a heterogeneous distribution. The lamellar-fault structures derive from twodimensional crystal nuclei.

The impurity in raw materials and the residual gas in working cells could produce a certain amount of impurities in the synthesis process of $\mathrm{cBN}$ crystals. The impurities adsorbed on the growing crystal surface prompt the lamellar-fault structures to form a big step under HPHT $[10,11]$. When the parallel steps were disturbed during the HPHT step growth process, the growth steps merged locally, as shown in Figure 3(b). The big steps on single crystal surfaces probably derive from dislocations. Because of the presence of some twisting defects and steps, the surface energy barrier of nucleation decreases; furthermore, cubic BN crystal nuclei have a higher possibility to grow around the twisting defects and big steps. The nucleation and growth mechanism transform the two-dimensional growth to dislocation growth mechanism.

The direction of all triangle-hole defects on (111) facets are pretty consistent relative to the edge of $\mathrm{cBN}$ single crystals, as shown in Figure 3(d). The reasons for the formation of triangle-hole defects are probably inclusions, vacancies, or dislocations. For (111)-B facets of cBN single crystals, $\mathrm{B}$ atoms have an $\mathrm{sp}^{3}$ empty orbit that can be stable; thus, (111)-B facets should be smooth surfaces. For (111)-N facets of cBN single crystals, $\mathrm{N}$ atoms have an $\mathrm{sp}^{3}$ hybridization orbit with a lone pair electron. Thus, it can easily bind with other atoms and form inclusions inside the cBN single crystals. The triangle-hole defects can be produced on the (111)-N facets of cBN single crystals when those inclusions cleaned out the surfaces of cBN single crystals effectively. When inclusions can exist in $\mathrm{cBN}$ single crystals under HPHT, some big vacancy loops were produced to relieve the internal stress of cBN single crystals [15]. The triangle-hole defects probably are the etch pits of edge dislocations exist on the surfaces of $\mathrm{cBN}$ single crystals when $\mathrm{cBN}$ single crystals were cleaned using the alkaline solutions.

\section{Conclusion}

The growth defects on (111) surface of cBN single crystals after rapid cooling are discussed for the first time by AFM technique. Some impurity particles, triangle-hole defects, lamellar-fault structures, and big steps were found on single crystal surfaces. A possible formation mechanism of several types of defects is revealed briefly. The analysis provides a better understanding for the growth mechanism of $\mathrm{cBN}$ single crystals under HPHT. The growth mechanism of cBN single crystals transforms the two-dimensional growth to dislocation growth mechanism under HPHT.

\section{Data Availability}

The data used to support the findings of this study are included within the article.

\section{Conflicts of Interest}

The authors declare no conflicts of interest.

\section{Authors' Contributions}

Lichao Cai conceptualized the study. Feng Jia and XingDong Yuan formally analyzed the manuscript. Lichao Cai was responsible for investigation. Bin $\mathrm{Xu}$ and Meizhe Lv were responsible for methodology. Bin $\mathrm{Xu}$ supervised the work. Lichao Cai wrote the original draft. Bin $\mathrm{Xu}$ and Meizhe Lv wrote, reviewed, and edited the manuscript.

\section{Acknowledgments}

This study was funded by the Natural Science Foundation of China (grant no. 51272139).

\section{References}

[1] W. Ding, B. Linke, Y. Zhu et al., "Review on monolayer CBN superabrasive wheels for grinding metallic materials," Chinese Journal of Aeronautics, vol. 30, no. 1, pp. 109-134, 2017.

[2] X. W. Zhang, "Doping and electrical properties of cubic boron nitride thin films: a critical review," Thin Solid Films, vol. 544, pp. 2-12, 2013.

[3] R. Mohammad and Ş. Katırcioğlu, "The structural and electronic properties of $\mathrm{BN}$ and $\mathrm{BP}$ compounds and $\mathrm{BN}_{x} \mathrm{P}_{1-x}$ alloys," Journal of Alloys and Compounds, vol. 478, no. 1-2, pp. 531-537, 2009.

[4] X. L. Yuan, J. Niitsuma, T. Sekiguchi, M. Takase, and T. Taniguchi, "Characterization of p-n junction formed at the boundary of facets in cubic-BN using scanning electron microscope," Diamond and Related Materials, vol. 14, no. 1112, pp. 1955-1959, 2005.

[5] S. Nakano, H. Ikawa, and O. Fukunaga, "Synthesis of cubic boron nitride using $\mathrm{Li}_{3} \mathrm{BN}_{2}, \mathrm{Sr}_{3} \mathrm{~B}_{2} \mathrm{~N}_{4}$ and $\mathrm{Ca}_{3} \mathrm{~B}_{2} \mathrm{~N}_{4}$ as solventcatalysts," Diamond and Related Materials, vol. 3, no. 1-2, pp. 75-82, 1994.

[6] O. Fukunaga and S. Takeuchi, "Growth mechanism of cubic $\mathrm{BN}$ using $\mathrm{Li}_{3} \mathrm{BN}_{2}$ solvent under high pressure," International Journal of Refractory Metals and Hard Materials, vol. 55, pp. 54-57, 2016.

[7] Y. Du, Z. Su, D. Yang et al., "Synthesis of black cBN single crystal in hBN-Li $\mathrm{L}_{3} \mathrm{~N}-\mathrm{B}$ system," Materials Letters, vol. 61, no. 16, pp. 3409-3412, 2007.

[8] T. Taniguchi and S. Yamaoka, "Spontaneous nucleation of cubic boron nitride single crystal by temperature gradient method under high pressure," Journal of Crystal Growth, vol. 222, no. 3, pp. 549-557, 2001.

[9] T. Taniguchi and K. Watanabe, "Synthesis of high-purity boron nitride single crystals under high pressure by using BaBN solvent," Journal of Crystal Growth, vol. 303, no. 2, pp. 525-529, 2007.

[10] M. Lv, B. Xu, L. Cai, X. Guo, and X. Yuan, “Auger electron spectroscopy analysis for growth interface of cubic boron nitride single crystals synthesized under high pressure and high temperature," Applied Surface Science, vol. 439, pp. 780-783, 2018.

[11] L.-W. Yin, M.-S. Li, Z.-G. Gong, B. Xu, Y.-J. Song, and Z.-Y. Hao, "Analysis of nanometer inclusions in high pressure synthesized diamond single crystals," Chemical Physics Letters, vol. 355, no. 5-6, pp. 490-496, 2002. 
[12] X. Guo, B. Xu, W. Zhang, Z. Cai, and Z. Wen, "XPS analysis for cubic boron nitride crystal synthesized under high pressure and high temperature using $\mathrm{Li}_{3} \mathrm{~N}$ as catalysis," Applied Surface Science, vol. 321, pp. 94-97, 2014.

[13] Z. X. Wen, B. Xu, L. C. Cai, Y. C. Cai, Z. Wen, and M. Z. Lv, "Influence of particle size of $\mathrm{Li}_{3} \mathrm{~N}$ catalyst on synthetic effect of cubic boron nitride single crystal," Journal of Synthetic Crystals, vol. 43, pp. 285-288, 2014.

[14] H. Yamane, S. Kikkawa, and M. Koizumi, "High-and lowtemperature phases of lithium boron nitride, $\mathrm{Li}_{3} \mathrm{BN}_{2}$ : preparation, phase relation, crystal structure, and ionic conductivity," Journal of Solid State Chemistry, vol. 71, no. 1, pp. 1-11, 1987.

[15] L.-W. Yin, M.-S. Li, F.-Z. Li, D.-S. Sun, and Z.-Y. Hao, "Defect formation in diamond single crystals grown from the Fe-Ni-C system at high temperature and high pressure," Materials Research Bulletin, vol. 36, no. 13-14, pp. 2283-2288, 2001. 